\section{Influenza vaccination: lessons learned from the pandemic (H1N1) 2009 influenza outbreak}

\author{
PJM Openshaw ${ }^{1}$ and J Dunning ${ }^{1}$
}

From any perspective, the unfolding of a novel pandemic provides great challenges. It demands cooperation between scientists and clinicians in different disciplines, working under great pressure around the clock and around the globe. Decisions that are made have major and immediate implications that need to be communicated and acted on in time to make a difference. Normal conventions and processes are often set aside for the sake of keeping ahead of transmission. Once the outbreak is over, the urgent activity winds down but momentum needs to be maintained to take stock of what has been learned and to retain the capacity to gear up again in future outbreaks.

As we move from the winter to summer, there is now very little influenza activity in the Northern hemisphere. The pandemic of influenza A (pH1N1/09) that we witnessed last year continues to move around the globe, promising to establish itself as the dominant strain of seasonal influenza for some years to come. ${ }^{1}$ Although we cannot predict with certainty how it will behave, it seems possible that it will maintain some of its unusual characteristics: its propensity for infecting younger adults, for causing severe disease in children, asthmatics, the pathologically obese and pregnant women, and perhaps for avoiding those over 65 years of age.

So, what have we learned? First, pH1N1/09 is a generally mild infection, but in a small but significant minority of people it causes severe disease. Why it does this remains a puzzle, but the assumption is that in some individuals there is a combination of virus and host genotype that leads to either uncontrolled viral replication, enhanced lung viral burden and viral dissemination, or to an excessive host immune response (innate, mediator-mediated or cellular) that might be loosely described as an immunological storm. Perhaps a combination of enhanced viral replication and an overexuberant inflammatory response leads to the respiratory failure and sometimes multisystem disease that so devastated some infected persons.

The fact that many of those infected showed little or no disease does not mean that the pandemic had little impact on health or on health care (Figure 1). At the peak of the first and second waves of infection (in July and November 2009, respectively), the number of patients seeking medical care in south London and the west Midlands had the potential to overwhelm primary care, leading to the UK government's introduction of a telephone helpline to cope with the demand for advice and antiviral drugs in England. In the first week of November 2009, there were approximately 850 patients hospitalized with confirmed $\mathrm{pH} 1 \mathrm{~N} 1$, about $20 \%$ of whom required critical care. ${ }^{2}$ In the United Kingdom, influenza-associated bed-days increased from 4,163 in 2008 to 33,376 in 2009 , about a sevenfold rise.
In those aged 17-39 years, the increase was from 169 to 6,253 in the October to December periods, an extraordinary 37 fold increase. ${ }^{3}$ Many intensive care facilities were stretched to capacity. We must all be grateful that the worst-case scenarios that had to be planned for never materialized. We just about coped with what turned out to be a generally mild disease.

How do the events of 2009 impact on vaccine strategy? Up to last year, the normal seasonal flu vaccines were a trivalent preparation, offering protection against three strains of seasonal flu viruses (e.g., H1N1, H3N2, and B in 2009). The twice-annually selected vaccine viruses were grown in embryonated hens' eggs, chemically inactivated, and then further processed to produce the viral surface antigens for formulation into vaccine. ${ }^{4}$ Some companies (notably Novartis International AG, Basel, Switzerland and Baxter International Inc., Deerfield, IL) were moving from egg production to cell culture, and live attenuated mucosal influenza vaccine, FluMist (administered as a nasal spray) has been marketed by MedImmune, LLC (Gaithersburg, MD). FluMist is licensed in the United States, but not yet in Europe.

For European (EMEA) regulatory approval, seasonal flu vaccines need to show that they induce antibody-mediated immune responses measured on the serum of adult volunteers and meet thresholds that normally correlate with protection. ${ }^{5}$ However, the relationship between serological responses and protection is not linear or fixed and it is possible that the correlation that has been used for licensure over many decades only applies to conventionally formulated vaccines. New vaccines may break this correlation, either being highly protective but inducing poor serological responses or giving excellent serological responses,

${ }^{1}$ Centre of Respiratory Infection, National Heart and Lung Institute, Imperial College London, London, UK. Correspondence: PJM Openshaw (p.openshaw@imperial.ac.uk) 


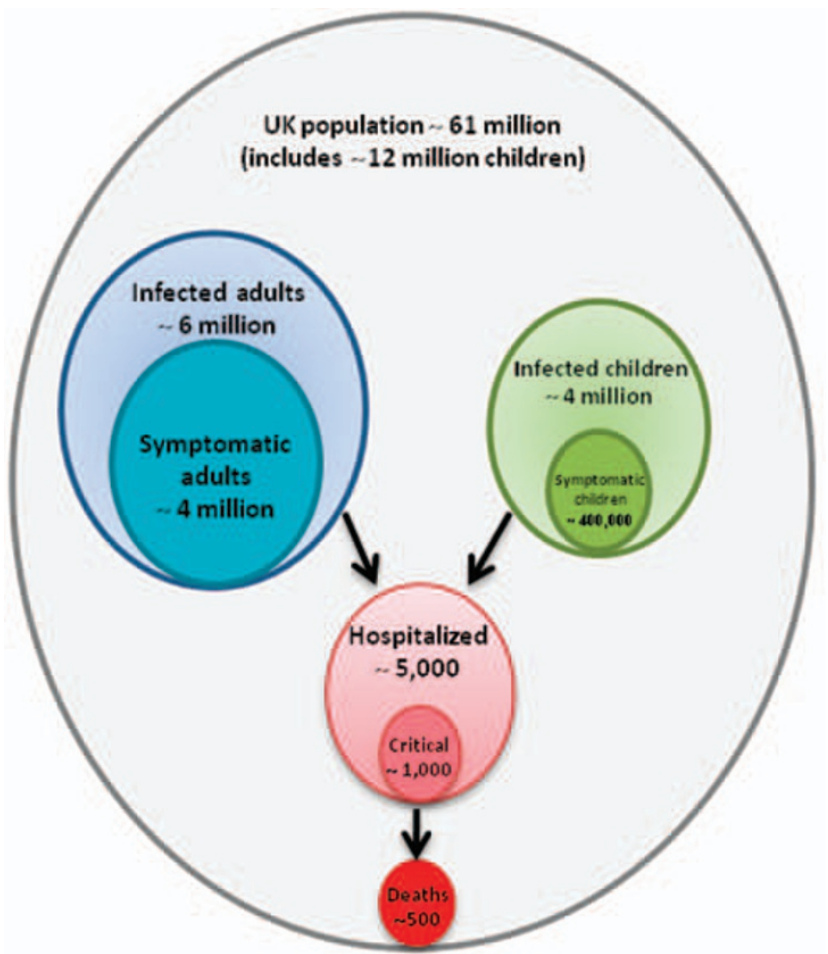

Figure 1 The estimated impact of pandemic (H1N1) 2009 influenza on the UK population.

meeting licensing requirements but offering no protection against infection, clinical influenza or the complications thereof. Mucosal vaccines, for example, might induce excellent local IgA-mediated protection but fail to induce a systemic neutralizing antibody response.

Other factors that are important to the protective effect of flu vaccines include the history of infection or vaccination. Historically, Mexico (one of the first countries to experience widespread disease) has one of the lowest influenza vaccination rates in the world at about $1 \%$ vaccine coverage. This compares to about $10 \%$ for Nordic countries and Japan; nearly $20 \%$ for Germany, France, the Netherlands, and the United Kingdom; and 25-35\% in the United States and Canada. ${ }^{6} \mathrm{~A}$ single dose of seasonal flu vaccine is usually all that is needed to give protection because most vaccine recipients have been exposed to similar viruses or vaccine antigens and are partially primed. Those who have not met similar flu antigens normally require two doses of vaccine, administered 4-6 weeks apart but in the $\mathrm{pH} 1 \mathrm{N1} / 09$ outbreak, Pandemrix (the adjuvanted vaccine from GlaxoSmithKline plc, Brentford, Middlesex, UK) produces a strong serological response after a single dose in many adults. ${ }^{7}$ Similarly formulated vaccine against $\mathrm{H} 5 \mathrm{~N} 1$ infection needed two or more doses to induce good responses. ${ }^{8}$

The use of adjuvants is a critical factor in many new vaccines. Pandemrix uses the oil in water adjuvant AS03 (monophosphoryl lipid A). Vaccines incorporating AS03 (e.g., against H5N1 influenza) have been given to many thousands of healthy adults without significant safety concerns having been identified, and have been administered to several hundred children without significant reactogenicity. Other adjuvants used by GSK include AS04 (aluminum salt and monophosphoryl lipid A) used in the successful human papillomavirus vaccine Cervarix (GlaxoSmithKline plc) and AS02 used in a candidate malaria vaccine and has been given to around 7,000 adults and 3,000 children below 18 months of age without safety concerns having been identified. AS02 contains monophosphoryl lipid A, QS21, and an oil-in-water emulsion. ${ }^{9}$

Cross-protection against other flu viruses does occur to some extent after natural flu infection, ${ }^{10}$ but has not been observed with conventional seasonal flu vaccines. Although vaccination against seasonal H1N1 virus does not appear to protect against $\mathrm{pH} 1 \mathrm{~N} 1 / 09$, it is possible that some of the newer vaccines, from, for example, Novartis, GSK, or MedImmune, may induce worthwhile heterotypic protection. One of the major development goals must include vaccines that can be used year on year without frequent redesign in response to viral mutation and that offer broad protection.

Soon after the WHO declared pandemic level 6 on 11 June 2009, the UK government completed negotiations for $132 \mathrm{mil}-$ lion doses of $\mathrm{pH} 1 \mathrm{~N} 1 / 09$ vaccine, ${ }^{11}$ more than enough for everyone in the United Kingdom to receive two doses. Although vaccine manufacturers succeeded in producing these novel vaccines in record time, the arrival of the first batches roughly coincided with the peak of the second wave. By this time the vaccines were targeted at about 17 million people who were deemed at greatest risk of disease, rather than targeting the entire population, and the epidemic was already in decline in the Northern hemisphere. Spontaneous infection has left many with natural immunity, leaving fewer people susceptible in some regions and risk groups. The uptake in some risk groups (particularly pregnant women and children aged 
6 months to 5 years) was hit by the perception that vaccination may have unspecified dangers and that $\mathrm{pH} 1 \mathrm{~N} 1 / 09$ disease was generally mild.

Targeting vaccination at those most at risk of transmitting disease, rather than targeting those at greatest risk of complications, may have a greater impact on community circulation of infection, and vaccination rates as low as $20 \%$ in key groups spreading infection may be enough to reduce spread in some mathematical models of community transmission. pH1N1/09 mainly spread among school children aged up to 18 years, but caregivers and health-care workers may also have a significant role in transmitting influenza. $^{12}$

Conventional trivalent seasonal influenza vaccines have an excellent safety record. Although an increased risk of Guillain-Barre syndrome was reported after the use of swine influenza vaccine in 1976 in the United States, the debacle was arguably political rather than vaccine related. ${ }^{13}$ Numerous studies have failed to show an increased risk of Guillain-Barre syndrome with later flu vaccines, including those for pH1N1/09, ${ }^{14}$ whereas Guillain-Barre syndrome remains a known complication of influenza infection.

The experience with vaccination against $\mathrm{pH} 1 \mathrm{~N} 1 / 09$ is likely to bring fresh approaches to vaccination against nonpandemic seasonal flu. The mold of the traditional trivalent vaccines has now been broken, the value and safety of adjuvants in flu vaccines has been shown, and the reliance on production in eggs has been called into question. The rate of development of live vector mucosal vaccines is likely to accelerate and the licensing criteria need to be refined, with redoubled efforts to develop novel approaches that confer broad protection against diverse strains of influenza and even against multiple species of respiratory pathogen.

This time, we coped. The experience gained in the last 12 months has, however, changed our understanding of influenza outbreaks and the way in which vaccines against influenza are developed and used. The incentives toward change and the potential benefits of global cooperation in this area are better now than they have been for many years, providing an exciting prospect of truly worthwhile developments in the next few years. Let us hope that we can do even better when the next pandemic arrives.

\section{DISCLOSURE}

PJMO has advised the Department of Health as a member of the Scientific Pandemic Influenza panel (SPI); he also serves on the Joint Committee on Vaccination and Immunisation (JCVI) subgroup on influenza and RSV prophylaxis, is a member of the UK Scientific Advisory Group in Emergencies (SAGE), is a member of European Scientific Working group on Influenza (ESWI), and is a member of the Department of Health Influenza Clinical Information Network (FluCIN). PJMO leads Mechanisms of Severe Acute Influenza Consortium (MOSAIC), which funds JD and is supported by the Wellcome Trust and Medical Research Council (UK).

C) 2010 Society for Mucosal Immunology

\section{REFERENCES}

1. World Health Organisation. Pandemic (H1N1) 2009-update 99, weekly update, http://www. who.int/csr/don/2010_05_07/en/index.html (accessed 05 May 2010).

2. Department of Health. Pandemic H1N1 (2009) Influenza: Chief Medical Officer's Statistical Update, 15 April 2010, http://www.dh.gov.uk/ prod_consum_dh/groups/dh_digitalasset/dh_ 115427.pdf (accessed 05 May 2010).

3. Hospital Episode Statistics (HES), The NHS Information Centre for health and social care. Provisional monthly HES topic of interest: influenza, http://www.hesonline.nhs.uk/Ease/ servlet/ContentServer;jsessionid=wagtnz7841
?sitelD=1937\&categorylD=1243 (accessed 05 May 2010).

4. Department of Health. Influenza In Immunisation Against Infectious Disease 3rd edn (Salisbury, D., Ramsay, M. \& Noakes, K., eds) 185-201 (The Stationery Office, London, 2006, ).

5. Note for Guidance on Harmonisation of Requirements for Influenza Vaccines. Committee for Proprietary Medicinal Products (CPMP). The European Agency for the Evaluation of Medicinal Products, Human Medicines Evaluation Unit. 12 March 1997, http://www.ema.europa.eu/pdfs/human/ bwp/021496en.pdf (accessed 05 May 2010).

6. Macroepidemiology of Influenza Vaccination (MIV) Study Group. The macro-epidemiology of influenza vaccination in 56 countries, 1997-2003. Vaccine 23, 5133-5143 (2005).

7. Roman, F., Vaman, T., Gerlach, B., Markendorf, A., Gillard, P. \& Devaster, J.M. Immunogenicity and safety in adults of one dose of influenza $A$ $\mathrm{H} 1 \mathrm{~N} 1 \mathrm{v} 2009$ vaccine formulated with and without ASO3A-adjuvant: preliminary report of an observer-blind, randomised trial. Vaccine 28, 1740-1745 (2010).

8. World Health Organization (WHO). Options for the Use of Human H5N1 Influenza Vaccines and the WHO H5N1 Stockpile. WHO Scientific Consultation, 1-3 October 2007. (WHO, Scientific Geneva, 2007).

9. Garçon, N., Chomez, P. \& Van Mechelen, M. GlaxoSmithKline adjuvant systems in vaccines: concepts, achievements and perspectives. Expert Rev. Vaccines 6 , 723-739 (2007).

10. Hancock, K. et al. Cross-reactive antibody responses to the 2009 pandemic $\mathrm{H} 1 \mathrm{~N} 1$ influenza virus. N. Engl. J. Med. 361, 1945-1952 (2009).

11. Department of Health. Agreements secured for pre-pandemic vaccine for the UK. 15 May 2009, http://www.dh.gov.uk/en/Publichealth/Flu/ Swineflu/DH 09924 (accessed 05 May 2010).

12. Chan, Y.J. et al. Seroprevalence of antibodies to pandemic (H1N1) 2009 influenza virus among hospital staff in a medical center in Taiwan. J. Chin. Med. Assoc. 73, 62-66 (2010).

13. Sencer, D.J. \& Millar, J.D. Reflections on the 1976 swine flu vaccination program. Emerg. Infect Dis. 12, 29-33 (2006).

14. Pfeifer, D. \& Wood, D. Safety of pandemic vaccination. 6th WHO Meeting on Evaluation of Pandemic Influenza vaccines in Clinical Trials, Geneva, 18-19 February 2010. http://www. who.int/vaccine_research/diseases/influenza/ meeting_18_19Feb2010/en/index1.html (accessed 05 May 2010). 